\title{
Suspected early dementia
}

This article explores how imaging can be used to investigate a patient with suspected early dementia

\author{
Jonathan M Schott senior lecturer and honorary consultant neurologist ${ }^{1}$, Jason D Warren reader \\ and honorary consultant neurologist ${ }^{1}$, Frederik Barkhof professor of neuroradiology ${ }^{2}$, Martin N \\ Rossor professor of clinical neurology ${ }^{1}$, Nick C Fox professor of clinical neurology ${ }^{1}$
}

${ }^{1}$ Dementia Research Centre, Institute of Neurology, University College London, London WC1N 3BG, UK; ${ }^{2}$ Radiology and Image Analysis Centre (IAC), VU Medical Centre, Amsterdam, The Netherlands

This series provides an update on the best use of different imaging methods for common or important clinical presentations. The series advisers are Fergus Gleeson, consultant radiologist, Churchill Hospital, Oxford, and Kamini Patel, consultant radiologist, Homerton University Hospital, London. To suggest a topic for this series, please email us at practice@bmj.com.

A 58 year old right handed woman was referred with a three year history of word finding difficulty and poor memory. Problems with reading, calculation, and spelling had emerged more recently. She could find her way around and was still driving. She was more irritable and less outgoing. Her symptoms were slowly progressive with a suggestion of day to day variability. There was no significant past medical history and she took no medications. There was a family history of cardiovascular disease but not of dementia. She did not drink alcohol to excess but had a 25 pack year smoking history. The physical examination was unremarkable. The mini mental state examination score was 25/30: she could recall all three items after a delay, but lost points on orientation, naming, calculation, and for following a three stage command. Initial investigations, including full blood count, electrolytes, liver function, thyroid function, erythrocyte sedimentation rate, vitamin B12, folate, and a chest $x$ ray, were normal.

\section{What is the differential diagnosis?}

As with many patients presenting with early cognitive problems, the differential diagnosis is wide. Anxiety and depression are associated with cognitive symptoms and they also complicate established dementia. Space occupying lesions including tumours, though unlikely, require consideration.

Dementia—acquired, progressive cognitive impairment sufficient to impair activities of daily living - is more typically encountered in older people, but is not uncommon at younger ages. ${ }^{1}$ In an ex-smoker with a family history of cardiovascular disease, vascular cognitive impairment, which can be associated with focal cognitive symptoms, depression, and insidious rather than stepwise cognitive decline, should be considered.

Alzheimer's disease is the most common neurodegenerative cause of dementia. The history of memory and executive decline in the context of fluctuations makes dementia with Lewy bodies possible, even without parkinsonism or visual hallucinations. Naming and behavioural problems are commonly seen within the frontotemporal lobar degeneration spectrum, which includes behavioural variant frontotemporal dementia (previously Pick's disease), progressive non-fluent aphasia, and semantic dementia (fluent speech with loss of word meaning).

Critically, distinguishing these causes from one another has implications for management, as acknowledged in UK national dementia guidelines. ${ }^{2}$ Surgery for an intracranial mass lesion is potentially curative, as is pharmacotherapy for depression. Even in the absence of disease modifying therapies, other dementia disorders still require specific treatment strategies, such as modification of vascular risk factors in vascular cognitive impairment, and acetylcholinesterase inhibition for Alzheimer's disease and dementia with Lewy bodies, for example. Accurate diagnosis facilitates prognosis and counselling, referral to appropriate services and voluntary organisations, applications for relevant benefits, and important lifestyle, occupational, and legal decisions, including those related to driving.

\section{How can brain imaging help?}

Although a detailed clinical assessment remains the mainstay of the evaluation of a patient with early dementia, UK, European, and US guidelines recommend that all patients with cognitive impairment should undergo structural imaging as part of the diagnostic work up. ${ }^{345}$ Brain imaging is increasingly used to help distinguish different forms of dementia from one another, whereas in the past it was principally undertaken to exclude treatable intracranial mass lesions, haematomata, and 


\section{Learning points}

All patients with suspected dementia should have structural brain imaging

Structural imaging is useful not only for excluding intracranial space occupying lesions, but also for arriving at a specific dementia subtype diagnosis

The pattern of regional brain atrophy-particularly using high resolution volumetric magnetic resonance imaging (MRI) - has value in distinguishing the common neurodegenerative causes of dementia

T2 weighted and fluid attenuated inversion recovery (FLAIR) MRI sequences are highly sensitive to ischaemic damage in the cerebral white matter

Other MRI sequences and imaging modalities (including positron emission tomography (PET) and dopamine transporter (DAT) scans) have diagnostic value in particular clinical settings.

hydrocephalus, which account for less than $1 \%$ of all cases of dementia. $^{3}$

\section{Computed tomography}

Where magnetic resonance imaging (MRI) is not available or contraindicated, computed tomography (which does involve exposure to ionising radiation) can usefully exclude major space occupying lesions, hydrocephalus, and large infarcts. Modern multidetector computed tomography scanners make it possible to acquire volumetric imaging data of the whole brain in a few seconds, which allows high resolution multiplanar reconstructions to be performed. Images reconstructed in the coronal plane allow detailed assessment of the medial temporal lobe structures(fig $1 \Downarrow$ ).

\section{Magnetic resonance imaging}

MRI, with its superior contrast resolution, is increasingly preferred over computed tomography in the evaluation of suspected dementia. ${ }^{2} \mathrm{~A}$ basic dementia sequence including a high resolution structural volumetric $\mathrm{T} 1$ weighted scan and $\mathrm{T} 2$ weighted or fluid attenuated inversion recovery (FLAIR) sequences can be obtained in approximately 20 minutes. MRI does not involve ionising radiation, but claustrophobia may be a limiting factor in some patients. MRI is contraindicated in patients with pacemakers and certain metallic implants, and earplugs should be worn to prevent the possibility of cochlear damage.

T1 weighted volumetric MRI scanning provides a very detailed assessment of brain structure, allowing for the assessment of the presence or absence and pattern of brain volume loss, ie, atrophy. When evaluating a patient with cognitive impairment it is particularly valuable to assess for medial temporal lobe atrophy on coronal reformats, either qualitatively or using simple rating scales. The presence of bilateral, symmetrical hippocampal atrophy distinguishes mild Alzheimer's disease from controls with approximately $80-85 \%$ sensitivity and specificity (fig $2 \mathrm{~A} \Downarrow$ ). ${ }^{46}$ Alzheimer's disease is also associated with relatively greater and more disproportionate hippocampal atrophy than dementia with Lewy bodies (fig 2B). ${ }^{7}$ In contrast, asymmetric temporal lobe atrophy with an anterior $>$ posterior gradient is at least $85 \%$ specific for frontotemporal lobar degeneration, ${ }^{8}$ and focal left inferior/anterior temporal lobe atrophy is highly suggestive of semantic dementia (fig 2C). ${ }^{9}$ The presence of medial temporal lobe atrophy in patients with isolated memory impairment (mild cognitive impairment) has high predictive value for the subsequent development of Alzheimer's disease,${ }^{10}$ and this has been incorporated into new proposed diagnostic criteria. ${ }^{11}{ }^{12}$ However, the absence of medial temporal lobe atrophy does not exclude a diagnosis of Alzheimer's disease, and patients with young onset Alzheimer's disease may have prominent posterior atrophy with relative sparing of medial temporal lobe structures.

T2 weighted or FLAIR sequences are highly sensitive for detecting white matter abnormalities, which can reflect demyelination but, much more commonly in this age group, cerebrovascular disease. As well as detecting major strokes, these sequences allow visualisation of small strategic infarcts (such as within the thalamus and other subcortical nuclei) and small vessel ischaemic white matter damage. Although an increased white matter lesion load suggests vascular disease, particularly in combination with lesions in the basal ganglia and brain stem, the pathophysiology and cognitive consequences of MRI white matter hyperintensities remain the subject of ongoing research, ${ }^{13}$ and it is important not to over interpret minor vascular disease that commonly accompanies ageing. Significant white matter and other ischaemic changes in the presence of hippocampal atrophy support a diagnosis of mixed vascular cognitive impairment/Alzheimer's dementia (fig 2D).

MRI scanning using a variety of additional sequences can provide other valuable diagnostic information. Thus, in the correct clinical context, the presence of temporal lobe signal change is suggestive of infection or inflammation (fig $3 \mathrm{~A} \Downarrow$ ); ${ }^{14}$ diffusion weighted imaging can help distinguish acute from chronic vascular disease, and the finding of neocortical or striatal abnormalities can aid the diagnosis of Creutzfeldt-Jakob disease (fig 3B) $;{ }^{15}$ and T2* sequences sensitive to iron deposition can demonstrate microhaemorrhages due to amyloid angiopathy (fig 3C) or vascular disease. ${ }^{16}$

\section{Metabolic and functional imaging}

Although metabolic or functional imaging is typically not performed routinely, it can provide valuable diagnostic information in certain circumstances. Where dementia with Lewy bodies is suspected, demonstration of central dopamine depletion using positron emission tomography (PET) (fig 4A $\Downarrow)$ or single photon emission computed tomography (SPECT) dopamine transporter (DAT) scanning has good diagnostic sensitivity and specificity (78\% and $90 \%$ in one study $\left.{ }^{17}\right)$, and is now included as a suggestive feature in diagnostic criteria. ${ }^{18}$ The demonstration of temporoparietal hypometabolism using fluorodeoxyglucose (FDG) PET or SPECT scanning supports a diagnosis of Alzheimer's disease over frontotemporal lobar degeneration (fig 4B); ${ }^{19}$ and in patients with personality or behavioural change in whom structural scanning is normal, the demonstration of focal frontal hypometabolism using FDG-PET scanning supports a diagnosis of frontotemporal lobar degeneration..$^{20}$ Currently research tools, new PET tracers appear to have very high sensitivity and specificity for detecting cerebral amyloid pathology (Fig 4C, D), and are likely to find clinical utility in the near future. ${ }^{21}$ All these techniques involve 
the injection of radioactive tracers, with a small exposure to ionising radiation.

\section{Outcome}

Our patient underwent MRI, which revealed no evidence of a space occupying lesion, and very minor vascular load. There was highly asymmetric left temporal lobe atrophy with an anterior>posterior gradient (fig 2C). Neuropsychometric testing confirmed profound anomia and semantic memory impairment. A diagnosis of frontotemporal lobar degeneration, semantic dementia variant was made. Treatment with acetylcholinesterase inhibition was not indicated ${ }^{2}$ the patient contacted the driving authorities and stopped driving; and was referred for speech and language therapy and to the Pick's Disease Support Group.

Contributors: JMS conceived and drafted the manuscript, which was revised by the other authors who have all approved the final version. JDW selected the patient. JMS is the guarantor.

Competing interests: All authors have completed the Unified Competing Interest form at www.icmje.org/coi_disclosure.pdf (available on request from the corresponding author) and declare: no support from any organisation for the submitted work; this work was undertaken at University College London Hospitals/University College London, which received a proportion of funding from the Department of Health's National Institute for Health Research Biomedical Research Centres funding scheme. The Dementia Research Centre is an Alzheimer's Research UK Coordinating Centre and has also received equipment funded by Alzheimer's Research UK. JMS is a UK Higher Education Funding Council for England clinical senior lecturer. JDW is a Wellcome Trust senior clinical fellow. NCF is a Medical Research Council senior clinical fellow. MNR and NCF are National Institute for Health Research senior investigators. The funders did not influence the writing of this review. JMS will shortly be commencing a study with AVID

Radiopharmaceuticals Inc. who manufacture florbetapir (fig 4); the authors report no other relationships or activities that have influenced the submitted work.

Provenance and peer review: Not commissioned; externally peer reviewed.

Patient consent obtained.

1 Harvey RJ, Skelton-Robinson M, Rossor MN. The prevalence and causes of dementia in people under the age of 65 years. J Neurol Neurosurg Psychiatry 2003;74:1206-9.
2 National Institute for Health and Clinical Excellence (NICE). CG42 Dementia: NICE guideline. March 2011. http://guidance.nice.org.uk/CG42/NICEGuidance/pdf/English. Scheltens P, Fox N, Barkhof F, De Carli C. Structural magnetic resonance imaging in the practical assessment of dementia: beyond exclusion. Lancet Neurol 2002;1:13-21.

4 Knopman DS, DeKosky ST, Cummings JL, Chui H, Corey-Bloom J, Relkin N, et al. Practice parameter: diagnosis of dementia (an evidence-based review). Report of the Quality Standards Subcommittee of the American Academy of Neurology. Neurology 2001;56:1143-53.

5 Hort J, O'Brien JT, Gainotti G, Pirttila T, Popescu BO, Rektorova I, et al. EFNS guidelines for the diagnosis and management of Alzheimer's disease. Eur J Neurol 2010;17:1236-48.

6 Duara R, Loewenstein DA, Potter E, Appel J, Greig MT, Urs R, et al. Medial temporal lobe atrophy on MRI scans and the diagnosis of Alzheimer disease. Neurology 2008;71:1986-92.

7 Burton EJ, Barber R, Mukaetova-Ladinska EB, Robson J, Perry RH, Jaros E, et al. Medial temporal lobe atrophy on MRI differentiates Alzheimer's disease from dementia with Lewy bodies and vascular cognitive impairment: a prospective study with pathological verification of diagnosis. Brain 2009;132:195-203.

8 Likeman M, Anderson VM, Stevens JM, Waldman AD, Godbolt AK, Frost C, et al. Visual assessment of atrophy on magnetic resonance imaging in the diagnosis of pathologically confirmed young-onset dementias. Arch Neurol 2005;62:1410-5.

9 Chan D, Fox NC, Scahill RI, Crum WR, Whitwell JL, Leschziner G, et al. Patterns of temporal lobe atrophy in semantic dementia and Alzheimer's disease. Ann Neurol 2001;49:433-42.

10 Fox NC, Schott JM. Imaging cerebral atrophy: normal ageing to Alzheimer's disease. Lancet 2004;363:392-4.

11 Dubois B, Feldman HH, Jacova C, Dekosky ST, Barberger-Gateau P, Cummings J, et al. Research criteria for the diagnosis of Alzheimer's disease: revising the NINCDS-ADRDA criteria. Lancet Neurol 2007;6:734-46.

12 Albert MS, Dekosky ST, Dubois B, Feldman HH, Fox NC, Dickson D, et al. The diagnosis of mild cognitive impairment due to Alzheimer's disease: recommendations from the National Institute on Aging -Alzheimer's Association workgroups on diagnostic guidelines for Alzheimer's disease. Alzheimers Dement 2011;7:270-9.

13 Inzitari D, Pracucci G, Poggesi A, Carlucci G, Barkhof F, Chabriat H, et al. Changes in white matter as determinant of global functional decline in older independent outpatients: three year follow-up of LADIS (leukoaraiosis and disability) study cohort. BMJ 2009;6;339:b2477.

14 Schott JM. Limbic encephalitis: a clinician's guide. Pract Neurol 2006;6:143-53.

15 Macfarlane RG, Wroe SJ, Collinge J, Yousry TA, Jäger HR. Neuroimaging findings in human prion disease. J Neurol Neurosurg Psychiatry 2007;78:664-70.

16 Cordonnier C, van der Flier WM. Brain microbleeds and Alzheimer's disease: innocent observation or key player? Brain 2011;134:335-44.

17 McKeith I, O'Brien J, Walker Z, Tatsch K, Booij J, Darcourt J, et al. Sensitivity and specificity of dopamine transporter imaging with 123I-FP-CIT SPECT in dementia with Lewy bodies: a phase III, multicentre study. Lancet Neurol 2007;6:305-13.

18 McKeith IG, Dickson DW, Lowe J, Emre M, O'Brien JT, Feldman H, et al. Diagnosis and management of dementia with Lewy bodies: third report of the DLB Consortium. Neurology 2005;65:1863-72.

19 Foster NL, Heidebrink JL, Clark CM, Jagust WJ, Arnold SE, Barbas NR, et al. FDG-PET improves accuracy in distinguishing frontotemporal dementia and Alzheimer's disease. Brain 2007;130:2616-35.

20 Kipps CM, Hodges JR, Fryer TD, Nestor PJ. Combined magnetic resonance imaging and positron emission tomography brain imaging in behavioural variant frontotemporal degeneration: refining the clinical phenotype. Brain 2009;132:2566-78.

21 Clark CM, Schneider JA, Bedell BJ, Beach TG, Bilker WB, Mintun MA, et al. Use of florbetapir-PET for imaging beta-amyloid pathology. JAMA 2011;305:275-83.

Cite this as: BMJ 2011;343:d5568

(c) BMJ Publishing Group Ltd 2011 


\section{Further reading}

- Neuroimaging in dementia. Barkhof F, Fox NC, Bastos-Leite AJ, Scheltens P, eds. Springer-Verlag, 2011

\section{Figures}

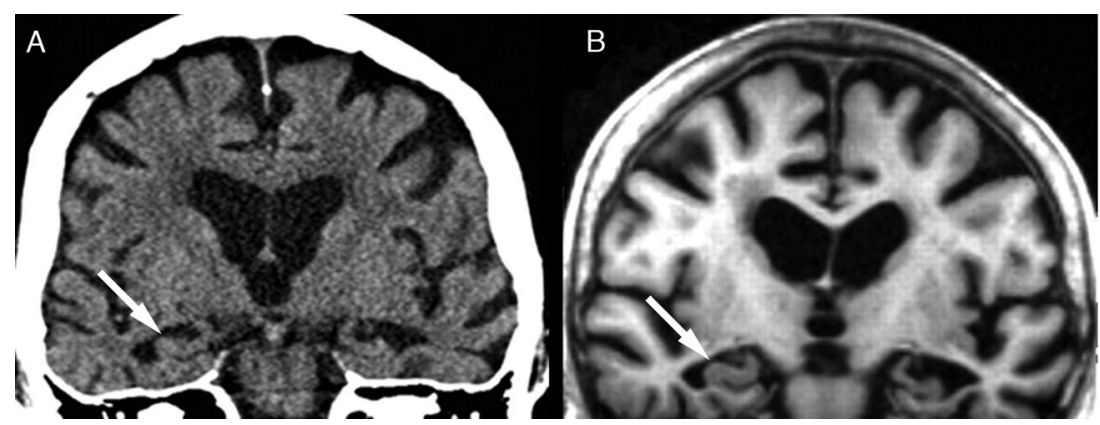

Fig 1 Bilateral medial temporal lobe atrophy (right hippocampus illustrated with arrows) in the same subject with Alzheimer's disease demonstrated on coronal images acquired with: (A) 64 detector row computed tomography scanning; (B) 1.5 tesla MRI volumetric T1 weighted sequence (adapted from Wattjes MP, Henneman WJ, van der Flier WM, de Vries O, Träber F, Geurts JJ, et al. Diagnostic imaging of patients in a memory clinic: comparison of MR imaging and 64-detector row CT. Radiology 2009;253:174-83, with permission)

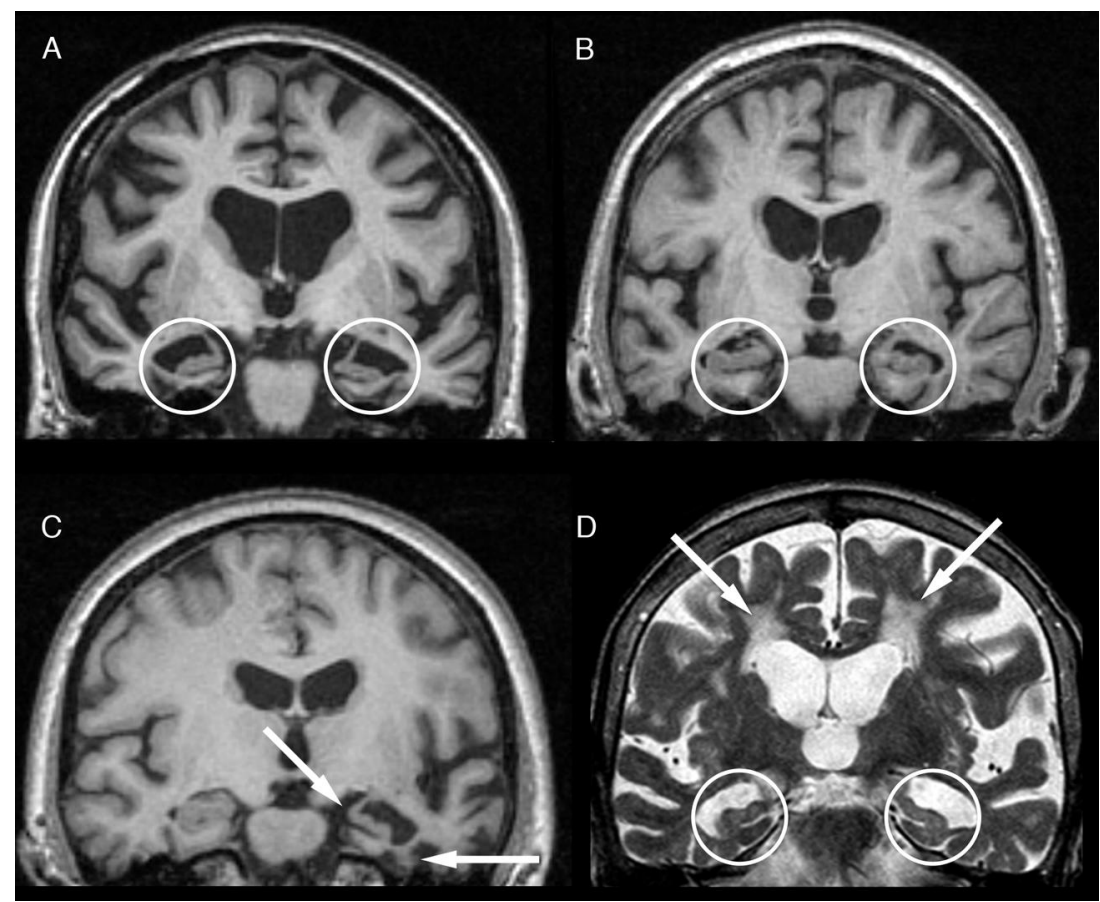

Fig 2 (A) T1 weighted coronal volumetric MRI at 1.5 tesla showing symmetrical medial temporal lobe (MTL) atrophy (circled) in postmortem proven Alzheimer's disease; (B) T1 weighted coronal volumetric MRI at 1.5 tesla showing relative sparing of MTL structures (circled) in postmortem proven dementia with Lewy bodies; (C) T1 weighted coronal volumetric MRI at 3 tesla showing highly asymmetric inferior left temporal lobe and hippocampal atrophy (arrows) in frontotemporal lobar degeneration, semantic dementia subtype; (D) T2 weighted coronal volumetric MRI at 1.5 tesla showing symmetrical MTL atrophy (circled) and small vessel white matter disease (arrows) in a patient with clinically diagnosed mixed vascular/Alzheimer's disease (D: adapted from Bastos Leite AJ, Scheltens P, Barkhof F. Pathological aging of the brain: an overview. Top Magn Reson Imaging 2004;15:369-89, with permission) 


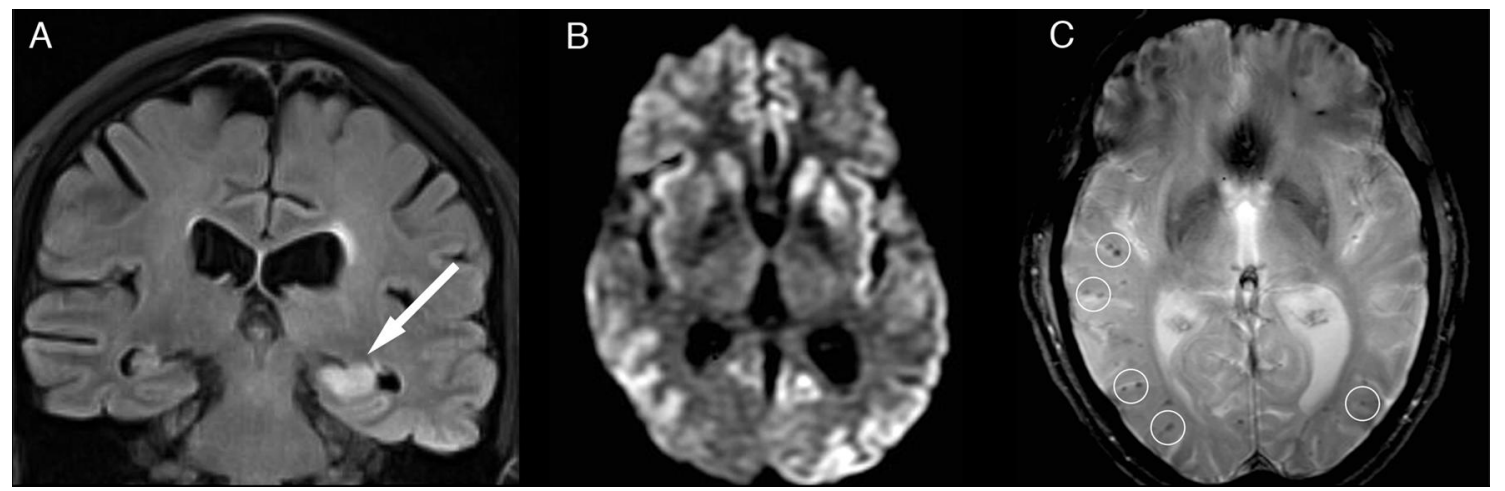

Fig 3 (A) Axial FLAIR MRI showing left hippocampal swelling and hyperintensity (arrow) in voltage gated potassium channel complex antibody associated limbic encephalitis; (B) Axial diffusion weighted MRI in a patient with sporadic Creutzfeldt-Jakob disease demonstrating widespread areas of confluent bilateral cortical restricted diffusion with similar restricted diffusion in the caudate nuclei and left putamen; $(C)$ axial gradient-echo T2* weighted imaging demonstrates multiple microbleeds (examples circled) indicating cerebral amyloid angiopathy in a patient with familial Alzheimer's disease (A: adapted from Barkhof F, Fox NC, Bastos-Leite AJ, Scheltens P, eds. Neuroimaging in dementia. Springer-Verlag, 2011, with permission; B: image courtesy Harpreet Hyare, MRC Prion Unit, University College London; C: adapted from Ryan NS, Bastos-Leite AJ, Rohrer JD, Werring DJ, Fox NC, Rossor MN, et al. Cerebral microbleeds in familial Alzheimer's disease. Brain 2011 : Jun 17, with permission)

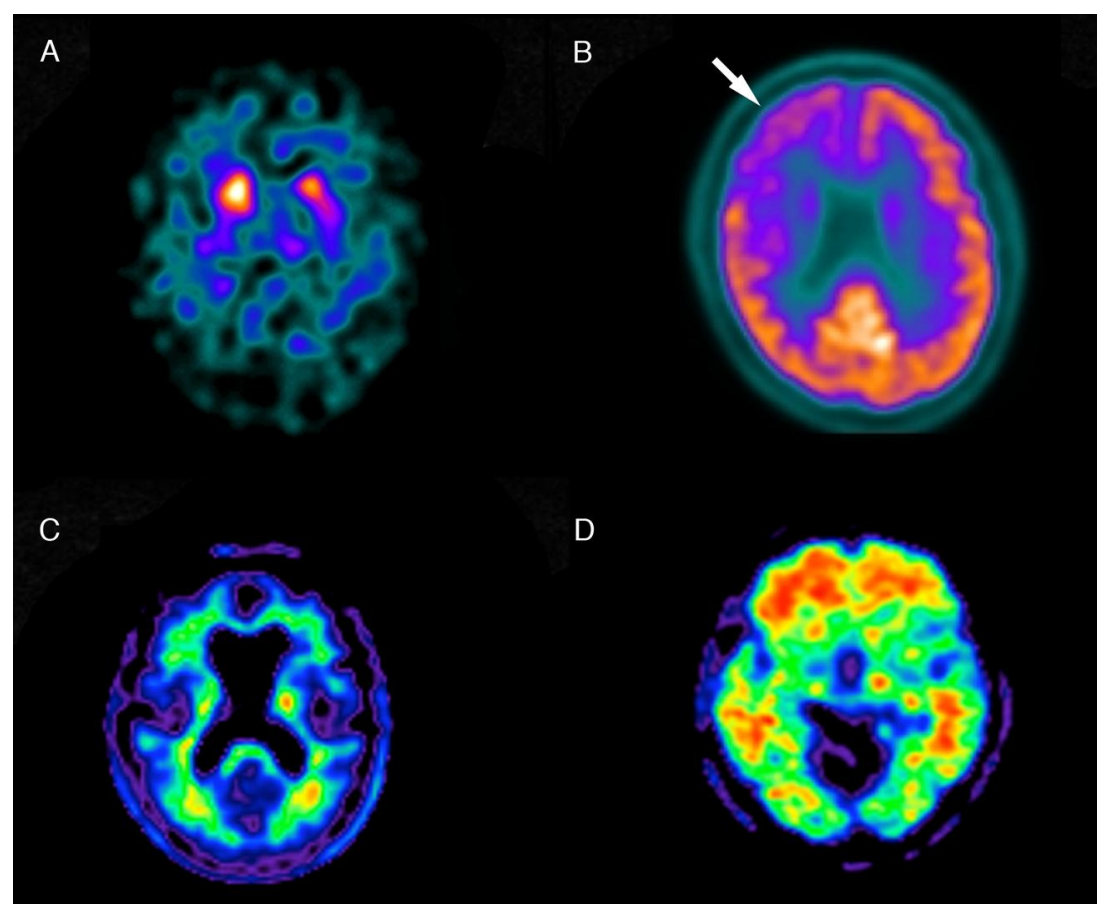

Fig 4 (A) Dopamine transporter imaging shows symmetrical reduced basal ganglia uptake (dot-like, rather than comma-like, in appearance) in a patient with clinically probable dementia with Lewy bodies; (B) FDG-PET shows right frontotemporal hypometabolism (arrow) in a patient with clinically probable behavioural variant frontotemporal dementia. Warm colours represent high glucose uptake; (C, D) F18 (florbetapir) amyloid PET imaging shows absence of significant binding in (C) a normal control; and (D) significant amyloid deposition (warm/hot colours) in a patient with clinically diagnosed Alzheimer's disease (images courtesy of AVID Radiopharmaceuticals, Inc.) 